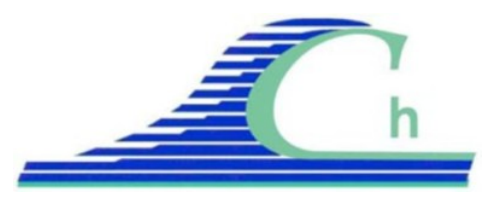

XII ${ }^{\text {èmes }}$ Journées Nationales Génie Côtier - Génie Civil

Cherbourg, 12-14 juin 2012

DOI:10.5150/jngcgc.2012.059-B（C) Editions Paralia CFL

disponible en ligne - http://www.paralia.fr - available online

\title{
Système de prélèvement en profondeur dynamique et en continu
}

\section{Pascal BAILLY DU BOIS ${ }^{1}$, Bertrand POUDEROUX ${ }^{2}$}

1. IRSN/PRP/SERIS/LRC - Institut de Radioprotection et de Sûreté Nucléaire, Pôle RadioProtection, Laboratoire de Radioécologie de Cherbourg - Octeville, rue Max Pol Fouchet, B.P. 10, 50130 Octeville, France.

pascal.bailly-du-bois@irsn.fr

2. Université de Caen, ESIX Normandie, Département GSI, Rue Louis Aragon, B.P. 78, 50100 Cherbourg-Octeville, France.

bertrand.pouderoux@unicaen.fr

\section{Résumé :}

L'acquisition de données et d'échantillons en profondeur à haute fréquence et en conservant la manœuvrabilité du navire représente un défi scientifique et technique.

Un système de prélèvement adapté à cet objectif a été développé. Il inclut trois composants :

- Une ligne de prélèvement permettant d'échantillonner 10 niveaux de profondeur simultanément ;

- Le DYNALEST, un lest dynamique de $80 \mathrm{~kg}$ permettant de maintenir une ligne immergée jusqu'à 50 mètres de profondeur, le navire restant manœuvrant entre 0 et 10 nœuds (5,1 $\mathrm{m} \mathrm{s}^{-1}, 15000 \mathrm{~N}$ de portance) ;

- Un automate permettant le prélever plus de 1200 échantillons à l'heure (10 niveaux toutes les trente secondes) et de les conditionner pour la mesure.

Ces matériels ont été testés en mer dans des conditions opérationnelles pour la collecte d'échantillons destinés à la validation de modèles 3D, à proximité de côtes et dans des zones à forts courants. 10 niveaux de profondeur ont été échantillonnés toutes les trente secondes jusqu'à 50 mètres de profondeur.

Les différents composants peuvent être utilisés associés ou séparément, selon les besoins. La principale application concerne le prélèvement d'eau en profondeur dans les zones proches des côtes, en présence de courants forts ou lors de prélèvements répétés et rapprochés. L'automate de prélèvement peut être utilisé pour tout type d'échantillonnage sur le terrain ou en laboratoire ; il est adapté pour gérer plusieurs lignes d'échantillonnage simultanées.

Mots-clés :

Prélèvement - Echantillonnage - Mer - 3D - Profondeur - Lest - Automate 


\section{Introduction}

Durant les campagnes océanographiques, les prélèvements en profondeur sont effectués habituellement à l'aide de bouteilles immergées, en parallèle sur une rosette télécommandée, ou en série sur une ligne. Cette technique nécessite l'arrêt du navire en station pendant le temps nécessaire à la manœuvre : au moins trente minutes pour chaque prélèvement pour les profondeurs inférieures à 50 mètres. Elle n'est pas envisageable pour réaliser des prélèvements à haute fréquence, dans les zones à forts courants ou à proximité des côtes.

Dans le cadre du projet DISVER, destiné à valider des modèles hydrodynamiques tridimensionnels à l'aide de mesures de radiotraceurs, nous devions collecter des échantillons à haute fréquence (toutes les 30 secondes), sur dix niveaux de profondeur simultanément. La zone de travail du Cap de La Hague est une des plus délicates à investiguer pour ce type d'étude. Elle associe de très forts courants (jusqu'à $5 \mathrm{~m} \mathrm{~s}^{-1}$, soit 10 nœuds), des côtes découpées avec de nombreux récifs et des profondeurs variant de 25 à $90 \mathrm{~m}$ en quelques kilomètres. Pour des raisons opérationnelles et de sécurité le navire doit être parfaitement manœuvrant en faisant route durant les prélèvements. Un système a été mis au point pour assurer les prélèvements en sécurité dans ces conditions.

La mise au point du système est décrite en distinguant ses trois principales composantes :

i. Une ligne de prélèvement permettant de prélever des échantillons à plusieurs profondeurs simultanément ;

ii. Un lest dépresseur (DYNALEST) permettant de maintenir une ligne immergée, le navire faisant route ;

iii. Un automate de prélèvement permettant de collecter des échantillons à haute fréquence.

\section{Matériel et méthodes}

\subsection{Ligne de prélèvement}

La ligne de prélèvement assure le pompage en continu de l'eau de mer à plusieurs profondeurs simultanément, le navire faisant route. Elle est constituée d'un faisceau de tuyaux dont chaque extrémité est immergée à une profondeur fixe. Plusieurs pompes assurent le pompage simultané sur tous les tuyaux pour amener à bord l'eau échantillonnée.

La ligne de prélèvement doit assurer simultanément plusieurs fonctions :

i. Immerger des tuyaux à la profondeur voulue pour échantillonner la masse d'eau traversée (de 20 mètres à 50 mètres de profondeur pour les configurations testées) ;

ii. Avoir un diamètre hydraulique le plus faible possible pour limiter la traînée de la ligne ; 


\section{XII ${ }^{\text {èmes }}$ Journées Nationales Génie Côtier - Génie Civil \\ Cherbourg, 12-14 juin 2012}

iii. Avoir un diamètre hydraulique suffisant pour prélever les volumes souhaités à la fréquence imposée, en assurant une séparation suffisante entre chaque échantillon (50 - 200 mL.mn-1 pour le cas présenté);

iv. Supporter les efforts mécaniques transversaux associés au déplacement par rapport à l'eau ;

v. Supporter le poids du lest qui la maintient immergée ;

vi. Supporter les capteurs enregistrant la profondeur d'immersion ;

vii. Transmettre en temps réel les mesures de profondeur sous le lest (capteur présenté figure 3) ;

viii.Etre facile à déployer et à relever à partir d'un navire faisant route.

Le nombre de niveaux, la profondeur et le débit de prélèvement peuvent être adaptés en fonction des besoins de chaque étude.

Le compromis entre les points 2 et 3 détermine le dimensionnement des tuyaux. Une section interne de $4 \mathrm{~mm}$ autorise un débit de $200 \mathrm{~mL} \mathrm{~m}^{-1}$ avec un tuyau de 100 mètres et une perte de charge de 0,5 bar, accessible avec une pompe péristaltique installée à bord du navire. Les pompes doivent être situées le plus près possible du niveau de l'eau pour limiter la pression hydrostatique associée à ce dénivelé. Des tuyaux en polyuréthane de section $4 \times 6 \mathrm{~mm}$ représentent un bon compromis de résistance, souplesse, transparence et maniabilité. Ils peuvent être raccordés par des raccords instantanés robustes. L'accouplement de dix tuyaux, du câble du sondeur (Ø $6 \mathrm{~mm}$ ) et du câble porteur de tuyaux (inox Ø 8mm) aboutit à une section moyenne de $20 \mathrm{~mm}$. Pour des raisons mécaniques et pour limiter la trainée, les tuyaux sont enveloppés dans une gaine en enroulant les tuyaux avec de l'adhésif fin (figure 1). Des informations concernant les calculs de trainée sont disponibles dans CHOI et al. (2011), MARK (2007), SUN et al. (2011)

Les tuyaux ne peuvent être enroulés directement sur le treuil du navire car ils seraient écrasés lors de l'enroulement : la force de traction exercée peut dépasser plusieurs tonnes. La ligne de prélèvement est accouplée sur un câble porteur qui supporte la traction du lest vers le bas et reprend régulièrement les efforts de traînée sur la ligne de prélèvement au moyen de mousquetons fixés à espacements réguliers (figure 2). 


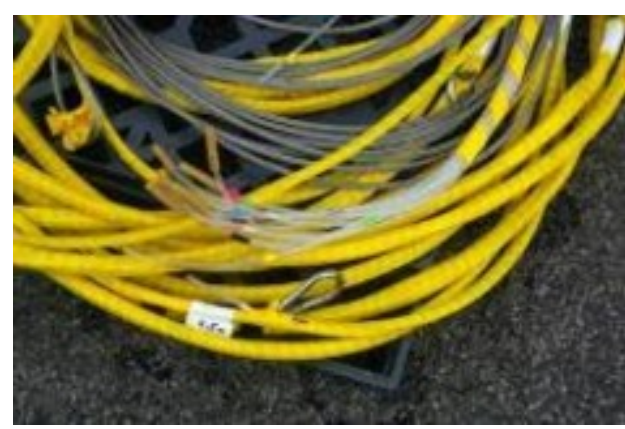

Figure 1. Ligne de prélèvement avec mousqueton de fixation et raccords largables vers les pompes

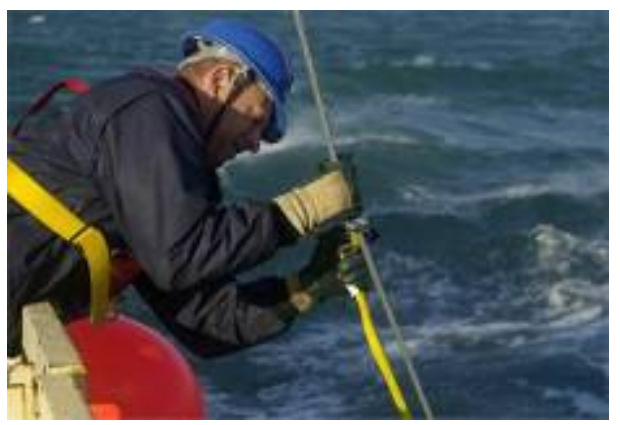

Figure 2. Fixation des tuyaux sur le câble porteur

Des capteurs de pression enregistreurs autonomes sont fixés régulièrement le long de la ligne pour connaître la profondeur réelle des prélèvements.

Caractéristiques de la ligne de prélèvement :

A. Câble porteur

- Acier galvanisé de 14 mm, charge de rupture de $90000 \mathrm{~N}$;

- Anneaux de fixation tous les 2,5 m (maillons de chaîne), fixés par serre câbles.

B. Ligne de prélèvement

- Câble inox de $8 \mathrm{~mm}$, charge de rupture de $30000 \mathrm{~N}$;

- Tuyaux polyuréthane de section $4 \times 6 \mathrm{~mm}, 1$ à 10 débouchant tous les 2,5 m (ligne de $30 \mathrm{~m}$ ) ou tous les 7,5 m (ligne de $100 \mathrm{~m}$ );

- 3 capteurs de pression répartis sur la ligne $\left(\mathrm{NKE}^{\circledR} \mathrm{SP} 2 \mathrm{~T} 100\right)$;

- Câble de données sondeur (Ø 6 mm) ;

- Mousquetons rapides tous les 2,5 m (Ø $10 \mathrm{~mm}$, rupture $1 \mathrm{t})$;

- Enveloppe en adhésif souple continue avec recouvrement entre chaque bande de $50 \%$;

- Raccords tuyaux et débitmètres ;

- Manille et émerillon.

\subsection{Lest dynamique - DYNALEST}

Un lest dépresseur ou lest dynamique est utilisé pour maintenir la ligne de prélèvement à la profondeur souhaitée avec une vitesse de déplacement du navire de 1 à 10 nœuds $\left(0,5-5 \mathrm{~m} \mathrm{~s}^{-1}\right)$, dans des conditions de manœuvre normales. La force verticale nécessaire est évaluée à $15000 \mathrm{~N}$ pour une profondeur de 30 à 50 mètres (calcul Mecaflux $^{\circledR}$ ). Le lest dépresseur utilise la vitesse de déplacement du navire pour créer une portance vers le bas qui augmente avec la vitesse du navire et est proportionnelle à la trainée du câble qui doit être maintenu immergé. Il en résulte que les efforts sont adaptés aux besoins et que l'engin peut être manœuvré par deux personnes lors de la mise à l'eau. 


\section{XII ${ }^{\text {èmes }}$ Journées Nationales Génie Côtier - Génie Civil \\ Cherbourg, 12-14 juin 2012}

Le tableau 1 présente les systèmes existants. La traînée représente la force qui s’oppose au déplacement du lest dans le sens du déplacement ; la portance est la force exercée par le lest vers le bas ; la finesse est le rapport entre la portance et la traînée, elle caractérise l'efficacité du lest. Ce critère est classiquement utilisé en aéronautique, en particulier pour qualifier les planeurs.

Tableau 1. Caractéristiques des lests dépresseurs existants.

\begin{tabular}{lllllll}
\hline $\begin{array}{l}\text { Fabriquant/ } \\
\text { revendeur }\end{array}$ & Type & $\begin{array}{l}\text { Portance } \\
\text { à } 10 \text { nouds }\end{array}$ & Poids air & Finesse & Largeur & Prix \\
\hline ANHYDRE & $\begin{array}{l}\text { V-FIN } 850 \\
\text { ScanFish } \\
\text { (BROWN et } \\
\text { al., 1996) }\end{array}$ & $11600 \mathrm{~N}$ & $195 \mathrm{~kg}$ & 5 & $2,17 \mathrm{~m}$ & $>15 \mathrm{k} \epsilon$ \\
EIVA & & $50 \mathrm{~kg}$ & $\approx 10$ & $1,60 \mathrm{~m}$ & $>15 \mathrm{k} \epsilon$ \\
IFREMER & Prototype & $\approx 10000 \mathrm{~N}$ & $200 \mathrm{~kg}$ & $4-9$ & $1 \mathrm{~m}$ & disponible \\
Plomb profilé & Lest passif & $10000 \mathrm{~N}$ & $1100 \mathrm{~kg}$ & 500 & $0,30 \mathrm{~m}$ & $>10 \mathrm{k} €$ \\
\hline
\end{tabular}

Aucun des systèmes existants ne combine un poids raisonnable, une portance et une finesse suffisantes pour l'objectif recherché, en particulier :

- Portance du lest supérieure à 10000 N à 10 nœuds.

- Manutention et mise en œuvre par deux personnes (masse inférieure à 100 kg) ;

- Rapport portance / traînée (finesse) supérieur à 50 ;

- Manœuvrabilité avec un navire faisant route normalement avec de fréquents changements de cap ;

- Sécurité du déploiement et en navigation

- Coût inférieur à $10 \mathrm{~K} €$;

Le profil adopté pour réaliser le DYNALEST est un profil dissymétrique de type NACA459. Le choix s'est fait, à l'aide du code Xfoil v.6.94 (http://web.mit.edu/drela/Public/web/xfoil/), sur des critères de robustesse afin de retenir un profil de portance et de finesse maximum, sans risquer des instabilités et des décrochements pour les vitesses et les incidences opérationnelles. Le risque de cavitation n’apparaît que pour des vitesses d'écoulement supérieures à 15 nœuds. Les dimensions retenues peuvent être adaptées aux besoins en modifiant la surface de l'aile (profondeur d'immersion, traînée de la ligne de prélèvement). Le matériau utilisé pour la fabrication de l'aile doit avoir une densité supérieure à celle de l'eau de mer pour ne pas flotter lors de la mise à l'eau, mais pas trop élevée pour qu'elle reste manœuvrable à la main. Il doit être suffisamment rigide pour supporter les efforts de portance, facile à mettre en forme, résistant à l'immersion saline et aux chocs. Le choix s'est porté sur l'aluminium massif qui satisfaisait à toutes ces exigences pour le moindre coût. Les profils ont été réalisés par usinage numérique sur la base des plans figure 3. 
Thème 3 - Instrumentation, mesures, imagerie et télédétection

Tableau 2. Caractéristiques retenues pour le DYNALEST (plans figure 3):

Profil porteur et empennage pour le réglage de l'incidence

Matériau : aluminium qualité marine (type $5083 \mathrm{H11}$ ); poids total : $80 \mathrm{~kg}$

Largeur : 1,6 m ; longueur : 2,2 m

Profondeur de travail : 20 à 100 mètres

Coût : $5700 €$

Aile

Profil NACA 459 (figure 3)

Largeur $1,6 \mathrm{~m}$

Corde $0,5 \mathrm{~m}$

Ailerons (2)

Profil NACA 009

Largeur $0,4 \mathrm{~m}$

Corde 0,2 $\mathrm{m}$

Gouverne de profondeur

Profil NACA 459

Largeur $0,8 \mathrm{~m}$

Corde 0,2 $\mathrm{m}$

\begin{tabular}{ll}
\hline Structure & Deux ronds en aluminium de $50 \mathrm{~mm}$ de diamètre. \\
\cline { 2 - 2 } & Longueur 2,35 $\mathrm{m}$ \\
\cline { 2 - 2 } $\begin{array}{ll}\text { Profilés aux extrémités } \\
\text { incidence de } 7^{\circ} \text { (calcul Xfoil) : }\end{array}$ & Portance $(P): 12350 \mathrm{~N}$ \\
\cline { 2 - 2 } & Traînée $(T): 147 \mathrm{~N}$ \\
\hline & Finesse $(P / T): 81$ \\
\hline
\end{tabular}

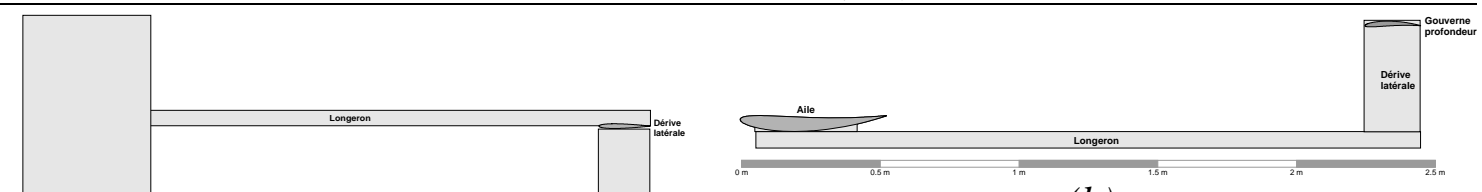

(b)

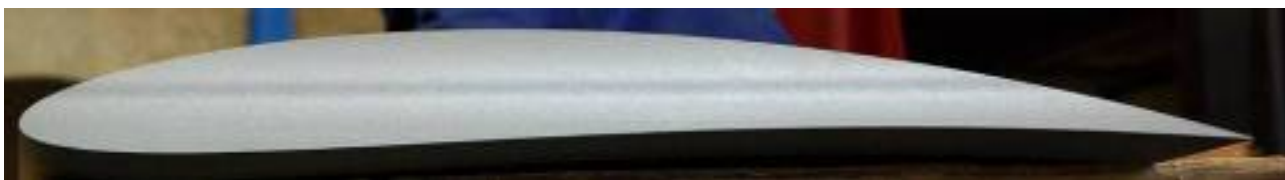

(c)

Figure 3. Plan du DYNALEST, $a$ : vue de dessus, $b$ : vue de côté, $c$ : profil NACA459 réalisé.

L'orientation de l'aile vis à vis de l'écoulement est réglable au moyen de la gouverne de profondeur. Celle-ci est située à 1,7 m de l'aile, elle peut tourner autour d'un axe longitudinal et impose un couple de rotation vertical réglable à l'aide d'une potence située sous la gouverne. 


\section{XII ${ }^{\text {èmes }}$ Journées Nationales Génie Côtier - Génie Civil \\ Cherbourg, 12-14 juin 2012}

Essais :

Des tests de portance et de manœuvrabilité ont été réalisés à l'aide d'une maquette au 1/15è et des tests de déploiement, de stabilité, de portance et de traînée dans le bassin de traction du centre IFREMER de Brest le système de fixation a pu être validé ainsi que le réglage de profondeur. Le DYNALEST a été utilisé en mer à bord du navire du CNRSINSU Côtes de la Manche lors de quatre campagnes en mer.

Le DYNALEST est manœuvrant jusqu'à 10 nœuds. Avec une longueur filée de 20 mètres, la traction instantanée mesurée sur le câble varie de pratiquement $100 \%$ autour de la valeur moyenne. Les pics mesurés atteignent $30000 \mathrm{~N}$, ce qui détermine le dimensionnement du câble de traction (rupture à $90000 \mathrm{~N}$ ).

Avec une longueur filée de 50 mètres, les variations de tension du câble s'atténuent et le comportement du DYNALEST est plus régulier. L'écart entre les pics de tension et la moyenne devient généralement inférieur à $50 \%$.

La figure 5 présente la comparaison entre la portance calculée et mesurée après diminution de l'angle d'incidence. La portance mesurée se situe dans la limite haute de celle qui avait été calculée ; elle correspondrait à une incidence de l'aile de 7 à $8^{\circ}$. La portance moyenne à 10 nœuds de $15000 \mathrm{~N}$ permet d'envisager des profondeurs de prélèvement supérieures à 50 mètres. Un angle d'incidence plus élevé a montré plus d’instabilités de la portance et un décrochage de l'aile lors d'un virage à 8 nœuds.

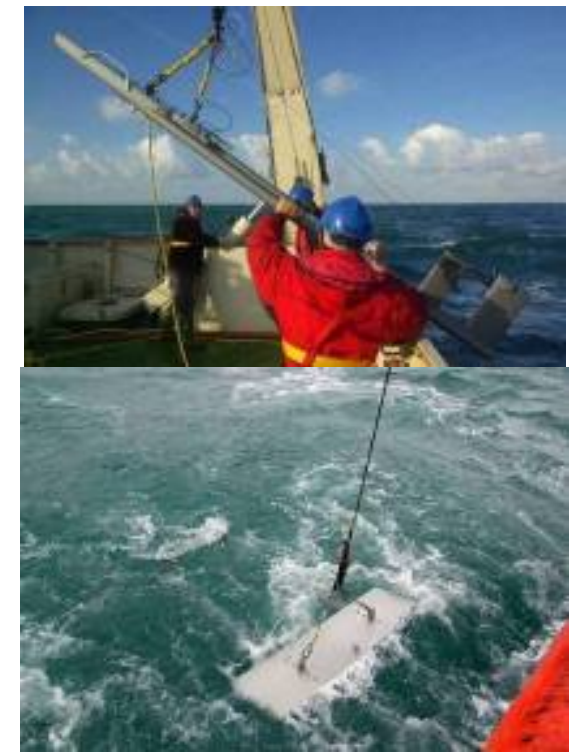

Figure 4. Mise à l'eau du DYNALEST.

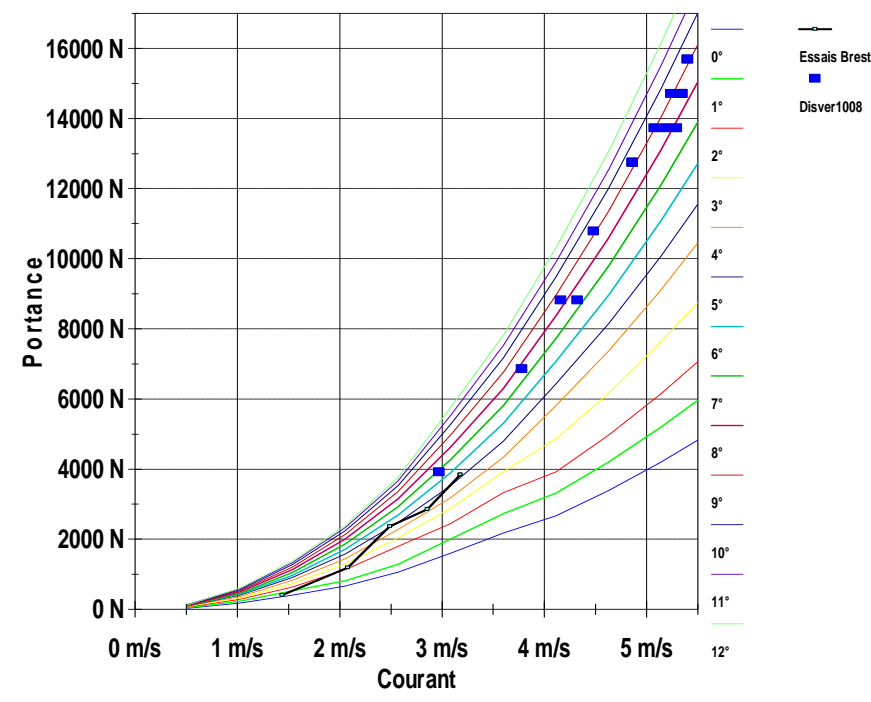

Figure 5. Portance calculée en fonction de l'angle d'incidence (légende à droite) et mesurée lors des essais en bassin et en mer. 


\subsection{Automate de Prélèvement à haute fréquence}

Le prélèvement simultané sur dix profondeurs toutes les 30 secondes (1200 échantillons à l'heure) ne peut pas être réalisé de manière manuelle. Il impose l'utilisation d'un automate de prélèvement dédié à cette tâche. L'IRSN s'est associé au laboratoire d'électronique de l'Ecole d'Ingénieurs de Cherbourg (EIC) pour développer un matériel adapté.

La figure 6 décrit les principales parties de l'automate. Le logiciel de commande enregistre l'heure, le débit et le volume sur chacune des voies et pour chaque échantillon. La position réelle du prélèvement est recalculée à postériori avec ces données, en tenant compte de la route du bateau, de la profondeur d'immersion de la ligne et du volume de chaque tuyau.

La circulation des échantillons dans les dix lignes de prélèvement est assurée par trois pompes péristaltiques, chacune pouvant supporter jusqu'à quatre lignes de prélèvement. Le contrôle des volumes prélevés est assuré par des débitmètres situés en amont de la table de prélèvement.

L'automate de prélèvement est un outil polyvalent, il a été utilisé pour réaliser des échantillonnages automatiques à la côte ou pour tout autre type de prélèvement séquentiel ou en série de liquides. Il a été utilisé lors de campagnes en mer pour réaliser des prélèvements réguliers durant plusieurs semaines d'eaux côtières, d'eaux de pluie ou de vapeur d'eau atmosphérique en pilotant en parallèle un système de déshumidification de l'air.

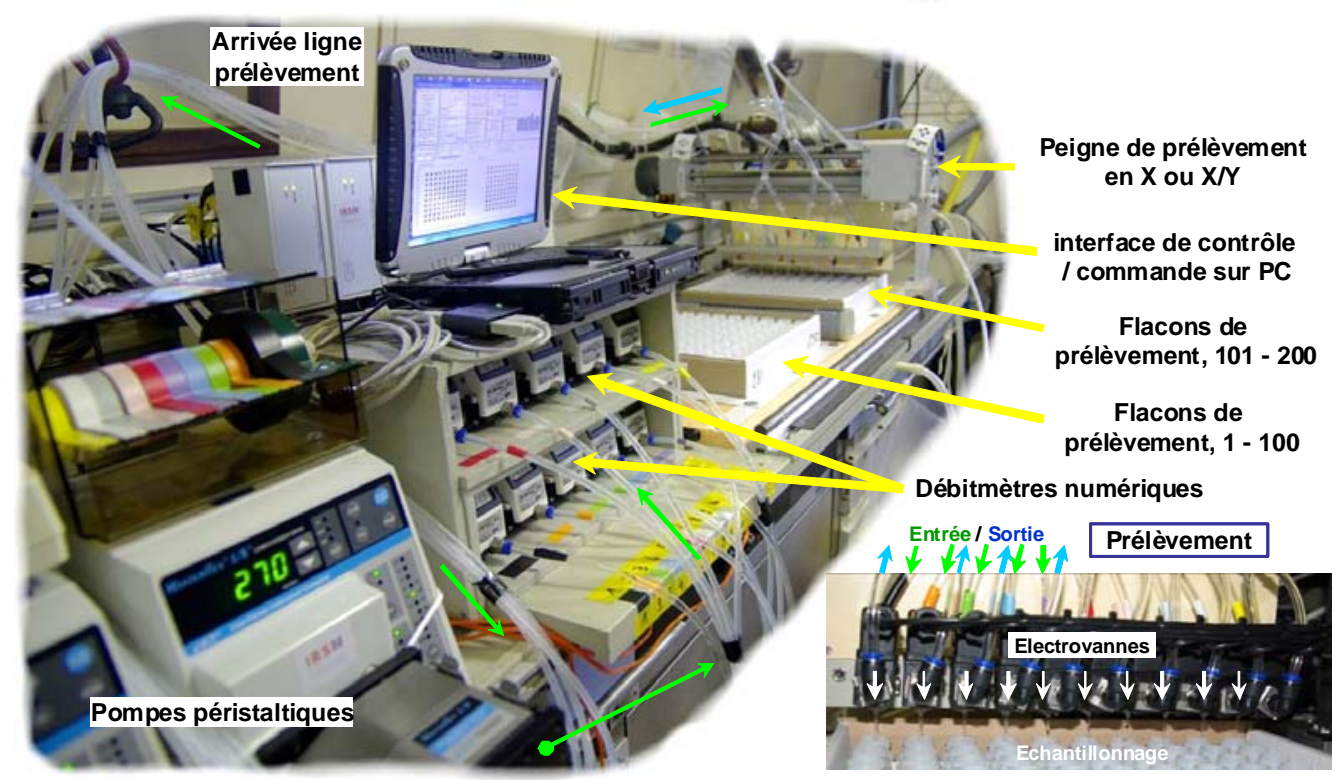

Figure 6. Automate de prélèvement à bord du Côtes de la Manche. 


\section{XII ${ }^{\text {èmes }}$ Journées Nationales Génie Côtier - Génie Civil \\ Cherbourg, 12-14 juin 2012}

\section{Résultats}

Le système de prélèvement a été mis en œuvre de manière opérationnelle depuis 2009 durant trois campagnes océanographiques, le navire a pu manœuvrer dans la zone du Cap de La Hague sans tenir compte de la ligne de prélèvement. Plus de 25000 échantillons ont été collectés en profondeur. Le contrôle de la distance entre le Dynalest et le fond est réalisé à l'aide du sondeur positionné sous le lest, qui fournit en continu au personnel de passerelle la distance entre le lest et le fond. Une distance minimum de cinq mètres a toujours été maintenue par sécurité. Le système a été utilisé avec une ligne de 30 mètres et une ligne de 70 mètres, elles ont permis de réaliser des prélèvements sur dix niveaux jusqu'à $25 \mathrm{~m}$ et 50 mètres de profondeur respectivement.

La figure 7 montre un exemple de résultats obtenus avec des mesures de tritium à proximité de l'émissaire de rejet de l'usine AREVA-NC. Les radiales présentées constituent des "coupes" transversales à l'axe de propagation du panache, à 850 mètres de l'émissaire. Environ 100 à 200 échantillons ont été collectés durant chaque radiale, qui sont espacées chacune de 5 à 10 minutes.

\section{Conclusion}

Le projet DISVER a pour objectif la validation, par des mesures in situ, de modèles de dispersion de substances dissoutes en mer en trois dimensions. L'acquisition de données en profondeur utilisables pour cette validation représente un défi scientifique et technique.

Il a été nécessaire de développer des outils spécifiques pour pouvoir prélever des échantillons en profondeur à haute fréquence, le navire faisant route :

- Le DYNALEST, un lest dynamique permettant de prélever jusqu’à 50 mètres de profondeur, le navire restant manœuvrant jusqu’à 10 nœuds ;

- Une ligne de prélèvement permettant d'échantillonner plusieurs profondeurs simultanément (10 dans l'application présentée) ;

- Un automate prélèvement permettant de collecter plus de 1200 échantillons à l'heure et de les conditionner pour la mesure.

Ces matériels ont été testés en mer dans des conditions opérationnelles pour le prélèvement d'échantillons destinés à la validation de modèles 3D plus de 20000 échantillons ont été collectés de cette manière.

Le système de prélèvement peut être utilisé dans toutes les situations nécessitant la collecte d'échantillons en milieu aquatique à haute fréquence et/ou en profondeur en conservant la manœuvrabilité du bâtiment support jusqu’à 10 nœuds ou $5 \mathrm{~m} \mathrm{~s}^{-1}$.

Bien que ce système a été conçu pour des conditions d'échantillonnage particulièrement difficiles, il se veut polyvalent et peut être utilisé en totalité ou en partie dans toutes les circonstances où des prélèvements ou des mesures répétitives et en profondeur sont nécessaires en mer, en rivière ou dans des lacs. La profondeur maximale testée avec 
l'appareillage décrit est de 100 mètres, des profondeurs supérieures pourraient être atteintes en adaptant les dimensions du lest dépresseur et de la ligne de prélèvement.

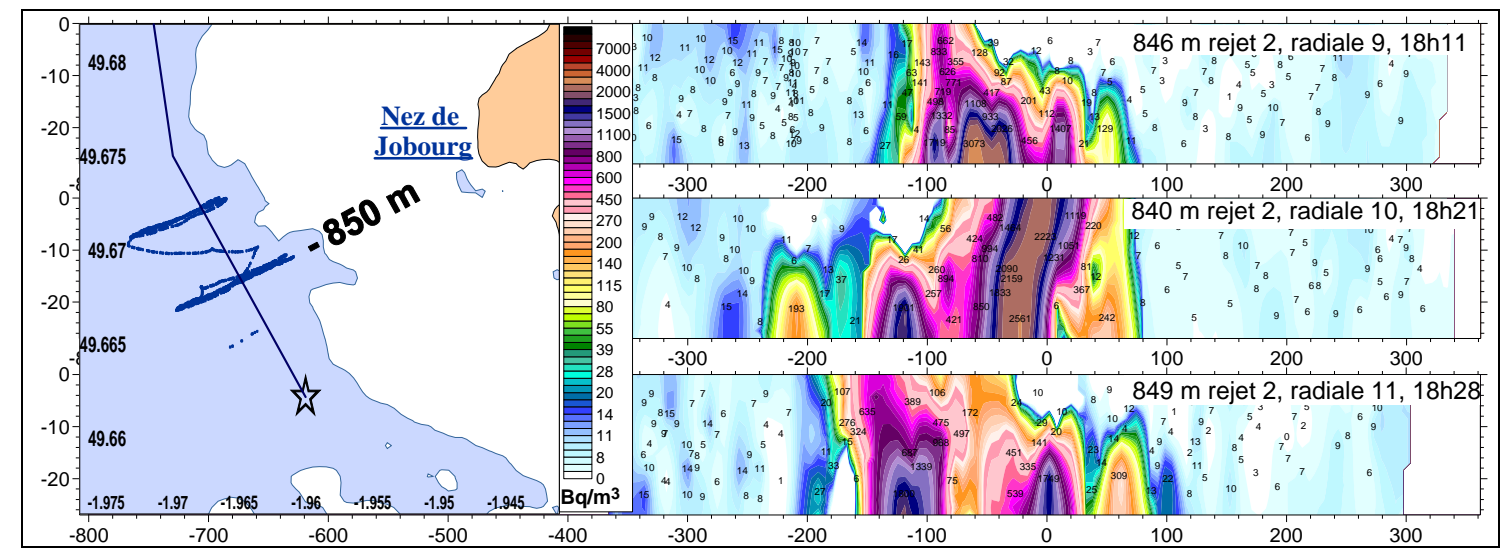

Figure 7. Concentrations en tritium mesurées durant la campagne Disver10, radiales 9 à 11 .

\section{Références}

BROWN J., BRANDER K.M., FERNAND L., HILL A.E. (1996). Scanfish: A high performance towed undulator. A new PC controlled towed undulator is aid to understanding coupling between physical and biological processes. Sea Technology, Vol. 37(9), pp 23-27.

CHOI J., SHIRAISHI T., TANAKA T., KONDO H. (2011). Safe operation of an autonomous underwater towed vehicle: Towed force monitoring and control. Automation in Construction Volume 20, Issues 8, pp 1012-1019. doi:10.1016/j.autcon.2011.04.002

MARK A.G. (2007). Transient behavior of towed cable systems during ship turning maneuvers. Ocean Engineering, Volume 34, Issues 11-12, pp 1532-1542. doi:10.1016/j.oceaneng.2007.01.002

SUN F., ZHU Z., LA ROSA M. (2011). Dynamic modeling of cable towed body using nodal position finite element method. Ocean Engineering, Volume 38, Issues 4, pp 529-540. doi:10.1016/j.oceaneng.2010.11.016 